\title{
Perfusion and thermal conductivity parameter extraction, from thermal imaging, for the quantification of the diabetic foot disease
}

\author{
by V. Serantoni ${ }^{*}$, F. Jourdan*, H. Louche* and A. Sultan** \\ * LMGC, Univ. Montpellier, CNRS, Rue St-Priest, 34095, Montpellier, France, vincent.serantoni@gmail.com \\ ** PHYMEDEXP, Univ. Montpellier, INSERM, CNRS UMR, CHRU Montpellier, Montpellier, France
}

\begin{abstract}
Diabetes Mellitus (DM) is a huge public health-care chronic disease issue, with about 425 millions of patients worldwide in 2017. DM is a serious pathology, especially through its complications and their severity, as one, called diabetic foot, which can lead to a lower limb amputation. The aim of the present study is to propose a method, based on the use of infrared imaging, to classify the foot state thanks to a bio-heat model. The protocol is based on a global cooling of the feet, the full thermal recording of the skin rewarming and on some parameters extraction.
\end{abstract}

\section{Introduction}

Among all the complications of DM, Diabetic Foot (DF) is one of special interest. DF can lead to the loss of sensitivity in the foot and to lower extremity ulcerations and infection. The relationship between DM and lower extremity amputation is well recognized [1]. The use of an infrared camera is common in the medical area and exists since many years [2].

Based on the important literature working on DF with thermal sensor, it seems clear that thermal information are valuable for the practitioner, but absolute temperature don't seem to be enough for an accurate classification of the foot state, transient information are needed [3]. Therefore, some works [4], [5] investigated the temporal thermal signal following a thermal stimulus. The return to equilibrium allowed to extract parameters and these parameters were then compared to the state of each patient. The principal conclusion of these works was that a difference between volunteers and patients with DF seemed to appear regarding a micro perfusion like parameter (generally denoted $\omega$ ), especially if this parameter is extracted using only the first seconds of the signal [5], [6]. Moreover, some of these works are able to obtain a good sensibility and specificity using a perfusion parameter evolving through time $(\omega(t))$. Results shown a high perfusion rate after the removal of the cold stimulus (reaction of the body), then a decrease of this parameter. In our opinion, this hypothesis may lead to good result but is physiologically wrong. Indeed, it is known that after a cold stimulus, blood perfusion is at the lowest level then increase slowly with the rewarming of the skin. Finally, in our knowledge, few or no article used the Penne's bio-heat equation, allowing even the conductivity parameter to change. The present paper will try to bring a physiological explanation of the thermal response following a cold stimulus.

Based on these remarks, a protocol, using infrared imaging during a transient rewarming following a cold stimulus beneath the foot, is proposed. A preliminary study allows to understand the sensibility of each parameter on the thermal response. Then, based on this knowledge, an experimental protocol is conducted in a hospital with a sample of patient with DF. A particular attention is paid to the post-processing (including calibration, correlation, ...) of the recorded thermal images [7]. An inverse method is then used for the parameters identification of the 1D Penne's bio-heat model. The perfusion and thermal conductivity parameters of this model are finally compared to the clinical classification of the patients.

\section{Materiel and methods}

The study includes 6 patients with diabetes (type 1 or 2) but no complication, 6 diabetic patients with neuropathy and/or arteriopathy and/or ulceration history, 2 healthy persons (control), for a total of 14 tested peoples.

All subjects were studied in an air-conditioned room maintained at a temperature between 21 and $23{ }^{\circ} \mathrm{C}$. Patients were asked to remove footwear and socks and to wait 5 minutes for the adaptation of their bare skin to indoor conditions. Subsequently, a cold stimulus was applied beneath the foot. The cold stimulus was a cooled plate $\left(10^{\circ} \mathrm{C}\right)$ applied beneath the foot during 30 seconds. The thermal response was then recorded during 5 minutes just after the removal of the stimulus. This stimulus enabled to extract a map of parameters under the feet and to visualize the support area of each patient. A microbolometer camera (Device-ALab, SmartIR640 640x512 pixels) was used for the thermal observations.

The bio-heat model used in the present work is based on the heat equation with a specific heat source for the blood micro-perfusion system, i.e.the Pennes's heat source [8] as shown in Eq. (1). Skin and soft tissue conduction are commonly low $\left(k<0.5 \mathrm{~W} \cdot \mathrm{m}^{-1} \cdot \mathrm{K}^{-1}\right)$. Thanks to the axisymetry of the thermal response around each valuated cooled point, a 1D model in the depth direction was chosen to analyse the skin surface temperature. Moreover, the model was supposed to be far enough from a vein or artery. The spatial domain in the thickness direction was limited to $5 \mathrm{~mm}$ deep 
(epidermis and dermis at feet level [9]), averaging the several layers under the skin surface to form an equivalent material noted tissue hereafter. The temperature $T(x, t)$ of the tissue in a spatial point located in the thickness position $x$ at time $t$ can be modelled by:

$$
\rho C \frac{\partial T(x, t)}{\partial t}-k \nabla^{2} T(x, t)=\rho_{b} C_{b} \omega\left(T_{b}-T(x, t)\right)
$$

where $k$ is the tissue thermal conductivity, $\rho$ the density, $C$ the tissue heat capacity, $\omega$ the blood perfusion rate (in $s^{-1}$ ) parameter and $T_{b}$ the inner body temperature. Indices $b$ stand for blood.

\section{Results and discussion}

Experiences have shown that, for a good fitting of the model onto the experimental results, it is mandatory to keep free the three parameters $k, \omega$ and $T_{b}$. The study of the influence of these parameters, on the thermal result of the model, reveals that a special care need to be taken during the parameter's identification. Indeed, perfusion parameter has a weak influence on the signal, conductivity parameter a stronger influence during the first $50 \mathrm{~s}$ of the signal and, blood temperature parameter has the stronger influence.
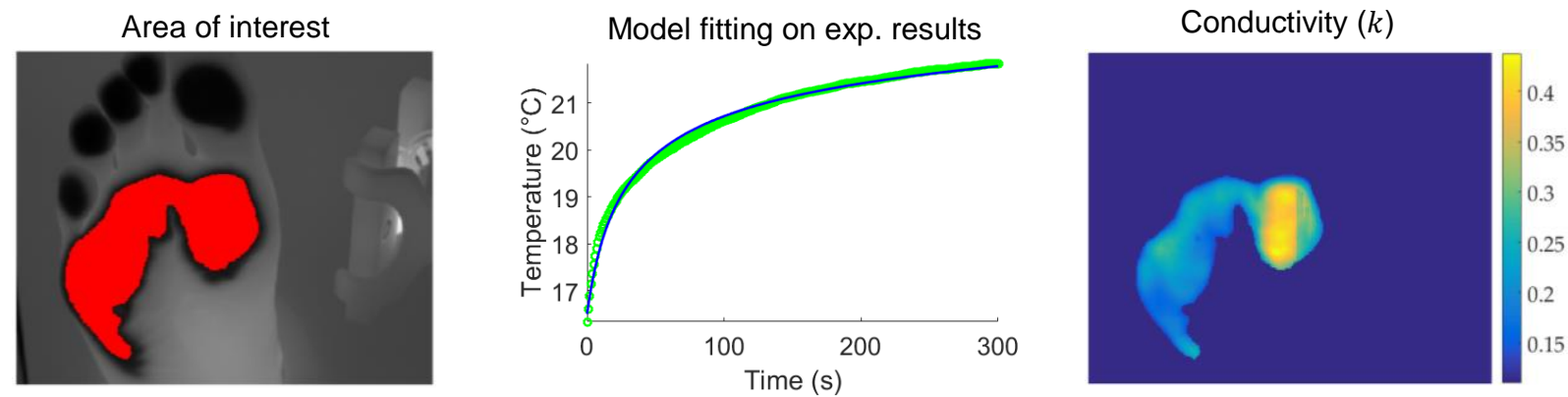

Figure 1: parameter's extraction on the area of interest. (left) Thermal image of the foot (in gray) after the removal of the cold plate; in red the selected area of interest. (center) Example of parameter extraction in one pixel. With the 3 identified parameter values, the model (in blue) fits well with the measure (in green). (right) Field of the thermal conductivity parameter $k$.

The identification of these three parameters was done with a steepest-descent algorithm, based on experimental data (figure 1). The obtained values were compatible with physiological values found in the literature for each terms. Results shown no correlation at all between the physiological state of the patient and the blood temperature, poor correlation with the perfusion parameter and a better correlation with the conductivity parameter.

The evaluation of the DF, through thermal analysis after cold stimulus, may be valuable with the conductivity parameter's characterisation but less with the constant perfusion Penne's parameter.

\section{Acknowledgment}

This work has been carried out with the support of the LabEx NUMEV (2011-LABX-076) into the I-SITE MUSE

\section{REFERENCES}

[1] D. G. Armstrong, A. J. M. Boulton, and S. A. Bus, "Diabetic Foot Ulcers and Their Recurrence," N. Engl. J. Med., vol. 376, no. 24, pp. 2367-2375, 2017.

[2] P. Sousa, V. Felizardo, D. Oliveira, R. Couto, and N. M. Garcia, "A review of thermal methods and technologies for diabetic foot assessment," Expert Rev. Med. Devices, vol. 12, no. 4, pp. 439-448, 2015.

[3] P. Soliz, C. Agurto, A. Edwards, and Z. Jarry, "Detection of diabetic peripheral neuropathy using spatial-temporal analysis in infrared videos," VisionQuest, 2016.

[4] G. Iven et al., "Non-contact Sensation Screening of Diabetic Foot Using Low Cost Infrared Sensors," in International Symposium on Computer-Based Medical Systems, 2014, pp. 479-480.

[5] V. Chekh, P. Soliz, M. Burge, and S. Luan, "A Physiological Thermal Regulation Model with Application to the Diagnosis of Diabetic Peripheral Neuropathy," in ACM International Conference, 2017, pp. 544-549.

[6] A. Bandini et al., "Effect of local blood flow in thermal regulation in diabetic patient," Microvasc. Res., vol. 88, pp. 42-47, 2013.

[7] V. Serantoni, F. Jourdan, H. Louche, and A. Sultan, "Proposal for a protocol using an infrared microbolometer camera and wavelet analysis to study foot thermoregulation," Quant. InfraRed Thermogr., 2019.

[8] H. H. Pennes, "Analysis of tissue and arterial blood temperatures in the resting human forearm. 1948.," J. Appl. Physiol., vol. 85, no. 1, pp. 5-34, Jul. 1998.

[9] C. Attinger, P. Cooper, and P. Blume, "Vascular anatomy of the foot and ankle," Oper. Tech. Plast. Reconstr. Surg., vol. 4, pp. 183-198, 1997. 